\title{
Estudio petromagnético y arqueomagnético del sitio El Cerrito de Los Agaves en la parte suroriental de los Altos de Jalisco, México
}

\section{Petromagnetic and acheomagnetic study of the El Cerrito de Los Agaves site in the southeastern part of the Jalisco highlands, Mexico}

\author{
Rodrigo Esparza López ${ }^{1}$, Verónica López-Delgado², Rubén Gejudo ${ }^{3, *}$, Avto Goguitchaichvili², \\ Teruaki Yoshida ${ }^{4}$, Mario Rétiz García ${ }^{1}$, Francisco Rodríguez Mota ${ }^{5}{ }^{\dagger}$, Miguel Gervantes-Solano ${ }^{2}$, \\ Juan Morales $^{2}$, Francisco Bautista ${ }^{3}$
}

${ }^{1}$ Centro de Estudios Arqueológicos, El Colegio de Michoacán, A.G., Cerro Nahuatzen 85, Col. Fracc. Jardines del Cerro Grande, 59370 , La Piedad, Michoacán, México.

${ }^{2}$ Laboratorio Interinstitucional de Arqueomagnetismo (Servicio Arqueomagnético Nacional), Instituto de Geofisica, Unidad Michoacán, UNAM. Antigua Carr. a Pátzcuaro No. 8701, Col. Ex Hacienda de Sán José de la Huerta, 58190, Morelia, Michoacán, México.

${ }^{3}$ Laboratorio Universitario de Geofísica Ambiental, Instituto de Geofísica y Centro de Investigación en Geografía Ambiental, Antigua Carr. a Pátzcuaro No. 8701, Col. Ex Hacienda de Sán José de la Huerta, 58190, Morelia, Michoacán, México.

${ }^{4}$ Universidad de Tokai, Shonan Campus: 4-1-1 Kitakaname, Hiratsuka-shi, Kanagawa 2591292, Japón.

${ }^{3}$ Proyecto Arqueológico Presa de la Luz, Cerro Nahuatzen 85, Col. Fracc. Jardines del Cerro Grande, 59370, La Piedad, Michoacán, México.

* Autor para correspondencia: (R. Cejudo) ruben@igeofisica.unam.mx

Cómo citar este artículo:

Esparza López, R., López-Delgado, V., Cejudo, R., Goguitchaichvili, A., Yoshida, T., Rétiz García, M., Rodríguez Mota, R., CervantesSolano, M., Morales, J., Bautista, F., 2021, Estudio petromagnético y arqueomagnético del sitio El Cerrito de Los Agaves en la parte suroriental de los Altos de Jalisco, México: Boletín de la Sociedad Geológica Mexicana, 73 (3), A2 10121. http://dx.doi.org/10.18268/ BSGM2021v73n3a210121

Manuscrito recibido: 15 de Diciembre de 2020

Manuscrito corregido: 14 de Enero de 2021

Manuscrito aceptado: 20 de Enero de 2021

La revisión por pares es responsabilidad de la Universidad Nacional Autónoma de México.

Este es un artículo Open Access bajo la licencia CC 1 -NC-SA (https://creativecommons.org/licenses/by-nc-sa/4.0/)

\section{RESUMEN}

En la actualidad la región de los Altos de Jalisco es todavía poco comprendida en cuanto a sus dinámicas culturales, principalmente lo relativo a los periodos de ocupación y filiación cultural. Hasta ahora se han identificado algunas relaciones con áreas culturales cercanas sobre todo con el Bajío y la Cuenca del río Lerma-Santiago. Gran parte de los asentamientos descubiertos en esta región pertenecen al periodo Epiclásico (600-900 d.C.), cuyo patrón de asentamiento se caracteriza con sitios establecidos en las mesetas, cerros o puntos elevados. En cuanto a su arquitectura, muchos de estos sitios están conformados por patios hundidos, plazas cerradas, además de palacetes con columnas, rasgos culturales que sugieren sociedades jerarquizadas con cierto control territorial. Uno de estos sitios que sobresale en esta región es el conocido como Presa de la Luz, el cual, a partir del año de 2012 ha sido objeto de un proceso de registro, prospección y excavación con el objetivo principal de reconocer el papel que jugó dentro de las dinámicas propias de Los Altos de Jalisco y su relación con las áreas circunvecinas. Adentro de este sitio se encuentra un complejo de edificios denominado como El Cerrito de Los Agaves, en el cual, durante los trabajos de investigación del año de 2020, se hallaron las huellas de pisos quemados dentro del Montículo Principal a 4 metros de profundidad, bajo el escombro. Estos pisos fueron fechados a través de la técnica de arqueomagnetismo. Los resultados apuntan a fechamientos cercanos al año 1000 d.C., presumiblemente, estas fechas corresponden al periodo de abandono del sitio, ya que son muy similares a los fechamientos realizados por la misma técnica en el sitio de Plazuelas (localizado a $105 \mathrm{~km}$ al oriente de este sitio). Además de las fechas, se relacionaron estos resultados con la arquitectura propia del sitio y con los materiales arqueológicos extraídos durante el proceso de excavación (principalmente la cerámica). Con ello, podemos concluir que esta región de los Altos de Jalisco estuvo dentro de las mismas dinámicas sociales regionales durante el Epiclásico.

Palabras clave: Arqueomagnetismo, Altos de Jalisco, arqueología, cronología, fechamiento arqueomagnético.

\section{ABSTRACT}

The Altos de Falisco region is still poorly understood in terms of its cultural dynamics. Extensive relationships have been identified with other nearby cultural areas, especially with the Bajio and the Lerma-Santiago River Basin. Much of the settlements so far discovered in this region belong to the Epiclassic period (600-900 AD) whose settlement pattern is similar with sites established on nearby plateaus. Many of these sites are build-up of sunken courtyards, closed squares, as well as palaces with columns that suggest hierarchical societies. One of these sites that stands out in this region is the Presa de la Luz extensively investigated since 2012. Our interest is to recognize the role and dynamics in context of Los Altos de Falisco zone and its relationship with other surrounding areas. Within the site, there is a complex of buildings called $E l$ Cerrito de Los Agaves, where series of deep (about $4 \mathrm{~m}$ ) burned floors were identified. Archaeomagnetic dating of apparently two independent floors provides age intervals around 1000 AD. Presumably, these dates correspond to the period of abandonment of the site since they are remarkably similar to the ages reported for the Plazuelas (Guanajuato) site (105 km east of this site). These results were also compared with the architecture of the place and the archaeological materials from excavation, mainly ceramics. It may be concluded that this part of the Altos de Jalisco belongs the same regional social dynamics during the Epiclassic.

Keywords: Archaeomagnetism, Altos de Jalisco, archaeology, chronology, archaeomagnetic dating. 


\section{Introducción}

Localizado en la porción Sureste de la región conocida como Los Altos de Jalisco, El Cerrito de Los Agaves es uno de los sitios arqueológicos con arquitectura monumental de gran importancia para la arqueología de los Altos de Jalisco dada su cercanía con las regiones circunvecinas con tradiciones culturales que influenciaron en la arquitectura, la religión y el comercio.

El Cerrito de Los Agaves está ubicado a unos 800 metros al norte de la comunidad conocida como La Luz, municipio de Jesús María, Jalisco y a unos 10 kilómetros (en línea recta) al noreste de la cabecera municipal (Figura 1). Este sitio es una de las tres áreas con vestigios prehispánicos que conforman el Proyecto Arqueológico Presa de La Luz, y es la única hasta el momento que cuenta con monumentos arquitectónicos. Está constituido en su área nuclear por un patio cerrado (Plaza Principal) de grandes dimensiones, cuenta con un con altar central y el patio es limitado al oriente por un montículo piramidal de aproximadamente 12 metros de altura, al sur por una gran plataforma rectangular que se eleva desde el piso del patio unos 4 metros (Plataforma Sur) y al poniente y norte por dos pequeños montículos unidos todos ellos por una banqueta.

Adyacente al talud oeste de la gran plataforma (Plataforma Sur) hay un patio hundido de menor tamaño que el principal, cerrado por sus cuatro lados al oriente por la Plataforma Sur, al norte por un montículo de poca altura, al sur por lo que parece ser otra plataforma de gran tamaño, pero de menor altura que la Plataforma Sur a la que se une por su costado sur y al poniente por un muro o banqueta que cierra el patio en su totalidad. Al sureste del área nuclear aproximadamente a 70 metros de distancia existe otro conjunto de dos montículos piramidales unidos por un patio abierto por sus costados oriente y sur. (Figuras $2 \mathrm{a}$ y $2 b)$.

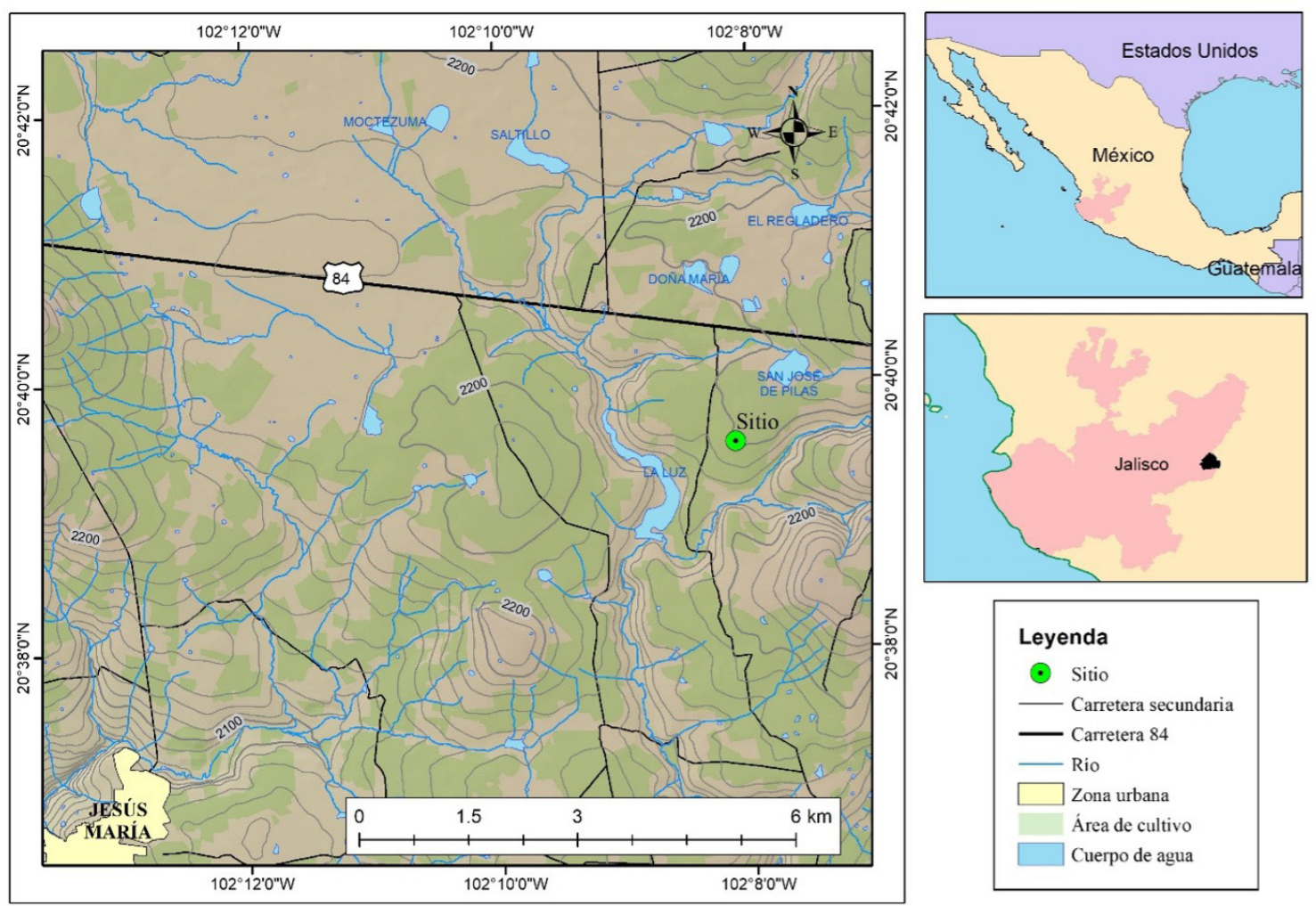


El inicio de los trabajos de investigación arqueológica de El Cerrito de Los Agaves se remonta al año 2017 en una primera temporada de 2 meses en campo en la que se realizaron los trabajos de topografía general, el trazo reticular del área de interés y la excavación de tres pozos de sondeo (Esparza, et. al., 2018).

En el Pozo 1, localizado fuera del patio hundido en su parte oriente, se pudieron determinar dos características importantes, la primera, un nivelamiento del terreno con rellenos de tierra roja para poder desplantar los edificios, y segundo, dos momentos de ocupación que se distinguían a par-

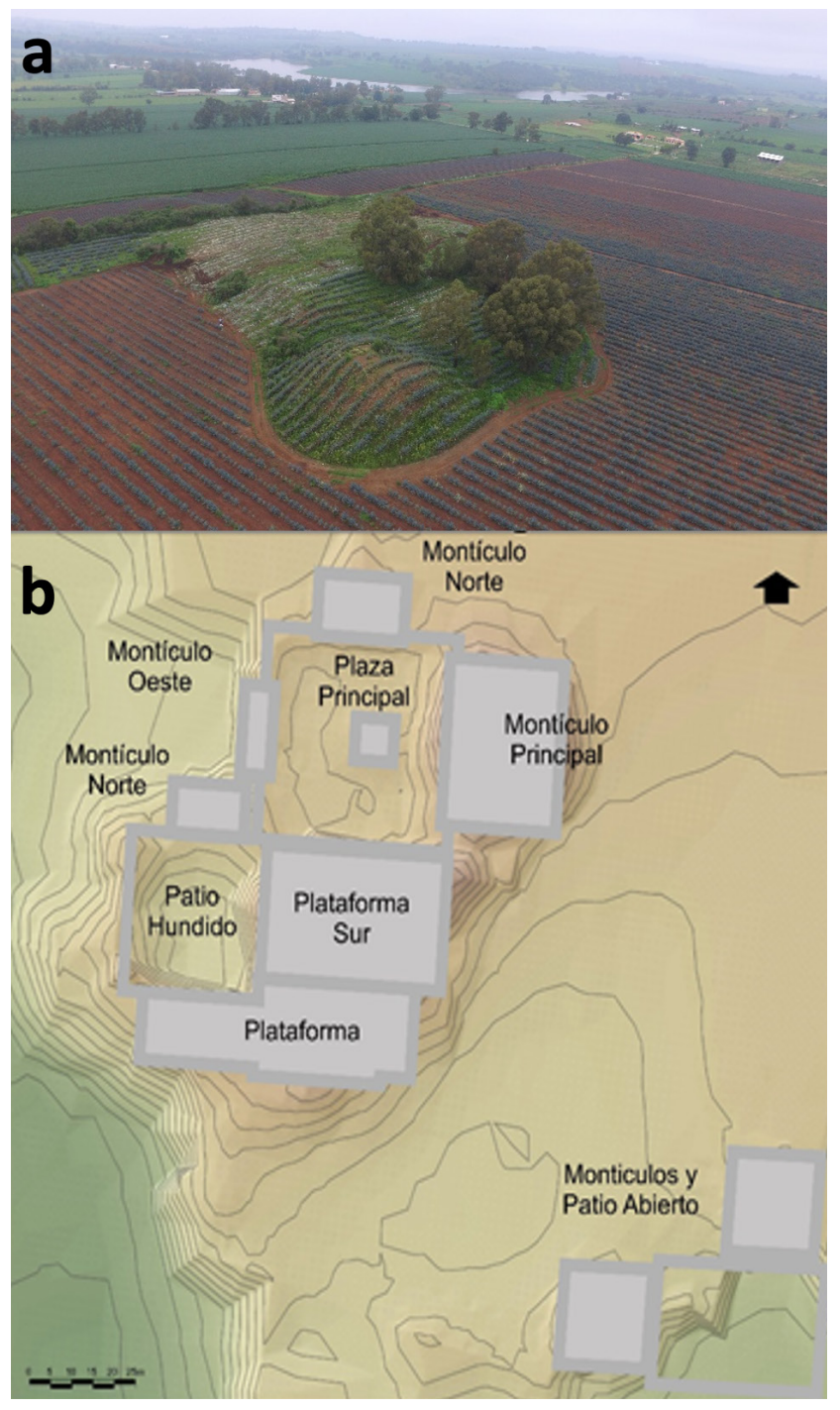

Figura 2 a) Vista general del sitio con el uso del dron. Fotografía: Cortesísa de Arqueodron. b) El levantamiento fotogramétrico. (Realizó César Hernández y Mario Rétiz). tir de la estratigrafía. De igual forma en el Pozo 2, ubicado dentro del patio, se observó la misma secuencia.

Lamentablemente los materiales cerámicos hallados en estos pozos fueron escasos como para establecer una seriación cronológica, sin embargo, fue posible establecer que la mayoría de los materiales pertenecen al periodo Epiclásico (600-900 d.C.) al compararlos con los hallados en otros sitios de los Altos, la región central de Jalisco y la región del Bajío como lo veremos más adelante. (Montejano, 2007; López Mestas y Montejano, 2003 y 2009; Fernández, 2016; entre otros).

Durante otra campaña realizada en el año 2018, se estudiaron dos áreas, una en el altar central con una cala de aproximación y después con calas de seguimiento de muros y dos pozos de sondeo, y una cala en el centro del altar y otra en la esquina noroeste.

En la otra área, el montículo principal se trazó y excavó una cala de aproximación a la estructura la cual permitió observar el apisonado del patio que une ambas construcciones, el montículo principal con el altar central, liberando parcialmente una escalinata de acceso a la estructura y que corresponde a la última etapa constructiva de la misma (Figura 3a y 3b). Durante la temporada de excavación realizada en los meses de febrero y marzo de 2020 se continuó liberando el altar central en su cara sur, se traza una nueva cala de aproximación hacia la Plataforma sur y se dio inicio a la excavación para conocer la arquitectura de la fachada norte de la misma.

En otro frente (Montículo principal) se trazó una cala de seguimiento perpendicular a la realizada en temporadas anteriores con la finalidad de conocer más sobre la arquitectura de la estructura. Solamente se excavaron dos pozos, el primero (S12E2) a 2 metros de distancia de la cala principal y sobre el talud del montículo, el segundo (S14E2) a 6 metros de distancia hacia el sur de la cala principal.

Es en el pozo denominado S12E2, donde se localizó parcialmente lo que suponemos es un sistema arquitectónico similar al talud tablero, aunque por el grado destrucción de ambos no es 
posible hasta ahora afirmarlo. Las muestras de piso quemado para la datación arqueomagnética son extraídas del muro inferior que delimita este cuerpo.

La razón principal por la cual se hacen las excavaciones en el complejo del Cerrito de Los Agaves es que las otras dos zonas de vestigios alrededor de este sitio están compuestas principalmente de concentraciones de Manifestaciones Gráfico Rupestres (MGR) o petrograbados, entre las que destacan las conocidas como cruces punteadas o pecked cross (Figura 4a y 4b), representaciones relacionadas con el conocimiento astronómico, cabe destacar que en este sitio existe una de las concentraciones más altas de este tipo de MGR solo después de Teotihuacán en el estado de México y Xihuingo-Tepeapulco en el estado de Hidalgo (Esparza et. al., 2016).

\section{Estudio arqueomagnético}

Las muestras de mano arqueomagnéticas fueron tomados dentro de Pozo S12E2, en una profundidad aproximada de 4 metros. Los pisos aparentemente quemados y separados por un grueso muro (Figura 5) obteniéndose muestras orientadas magnéticamente del piso interior como del exterior. Con la finalidad de identificar la mineralogía magnética presente en los materiales estudiados se realizaron experimentos de susceptibilidad magnética en función de temperatura (Figura 6). Las temperaturas de Curie de las curvas termomagnéticas se determinaron mediante el método de Prévot et al., (1983). Todas las muestras estudiadas provenientes del piso exterior muestran un comportamiento termomagnético inestable marcados por una clara irreversibilidad de curvas de calentamiento y enfriamiento. Sin embargo, se aprecia que una sola fase ferromagnética, probablemente titanomagnetita pobre en titanio está presente. No es el caso para las muestras de piso interior donde se observan claramente dos fases magnéticas durante el calentamiento. La fase observada entre $250^{\circ} \mathrm{C}$ y $350^{\circ} \mathrm{C}$ desaparece completamente después de calentamientos de hasta $600^{\circ} \mathrm{C}$ lo cual podría ser indicativo de la inversión de titanomaghemita y su transformación hacia magnetita (Dunlop y Özdemir, 1997; 2015). A cada muestra le fue medida la magnetización remanente natural (NRM por sus siglas en inglés) utilizando un magnetómetro de giro AGICO JR6A.

Posteriormente las muestras fueron desmagnetizadas mediante la aplicación progresiva de campos magnéticos alternos (AF por sus siglas en inglés) aplicando campos con valores pico de hasta 90 mT (Figura 7). La dirección de la magnetización remanente característica $(\mathrm{ChRM})$ de todas
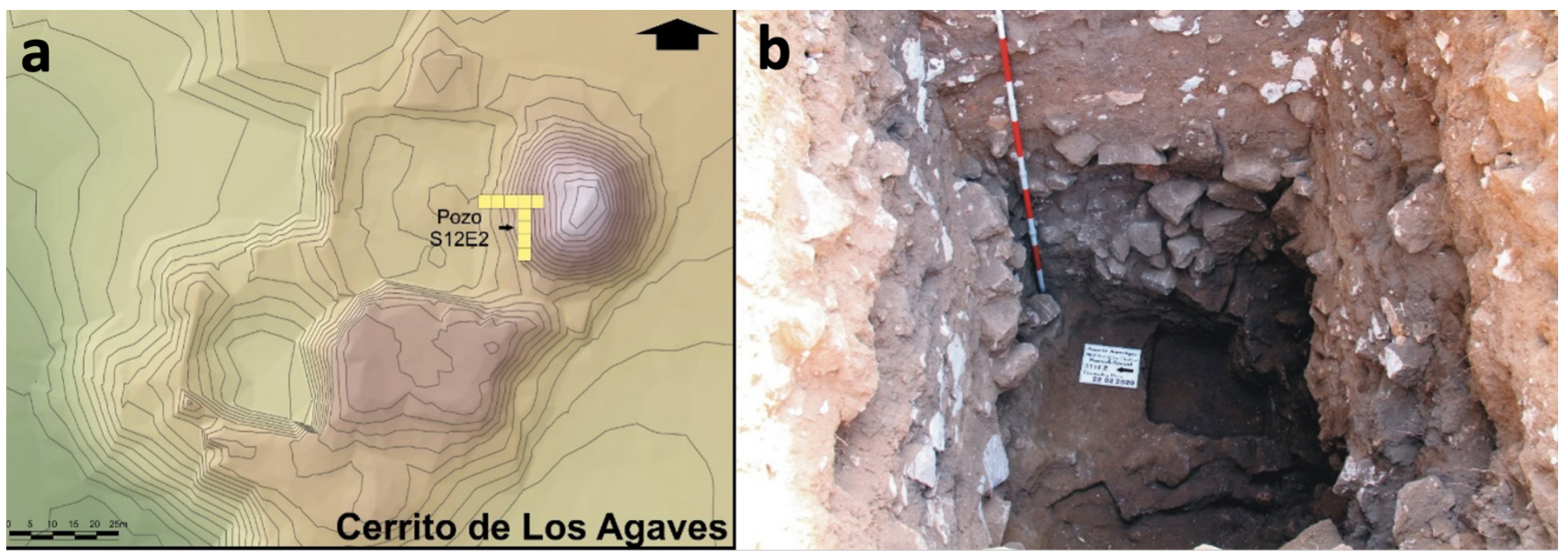

Figura 3 a) Localización del Pozo S12E2 del Montículo Principal. b) Vista superior del Pozo S12E2, con una profundidad de casi 4 metros. En la parte inferior es donde se tomaron las muestras para el fechamiento por arqueomagnetismo. 
las muestras se determinó mediante el análisis de componentes principales (Kirschvink, 1980) con al menos cuatro datos de los tratamientos magnéticos. Las direcciones medias para las muestras de piso se calcularon siguiendo la estadística de Fisher (1953) que incluyen el parámetro de precisión (k) y el ángulo de confianza (a95). En total se desmagnetizaron 20 muestras de ambos pisos quemados. En la figura 7 se muestran los ejemplos más representativos. Se aprecia esencialmente una única componente de magnetización con tendencia lineal al origen de coordenadas en los diagramas ortogonales lo cual indica que fue posible aislar las componentes características de las muestras. Las magnetizaciones secundarias de origen viscoso tienen una contribución poco significativa y son fácilmente removidas en las primeras etapas de desmagnetización con campos de $10 \mathrm{mT}$.

Aunque 20 especímenes fueron desmagnetizados mediante campos alternos, solo 15 de ellos (7 correspondiente a Piso Interior y 8 a Piso Exterior) mostraron una agrupación congruente y, por lo tanto, fueron considerados para el cálculo de paleodirecciones medias reportadas en la Figura 8. La datación arqueomagnética consiste en comparar estas direcciones medias con las que se esperan para la misma región y edad según el modelo geomagnético SCHA.DIF.14k (Pavón-Carrasco et al., 2014) utilizando el software archaeo_dating de Pavón-Carrasco et al., (2011). Los resultados del ejercicio de datación están reportadas en las figuras 9 y 10 .

\section{Discusión y conclusión}

Para entender la secuencia cronológica del sitio de Los Agaves, hay que recordar la cercanía que existe con otros sitios ya estudiados anteriormente por arqueomagnetismo, como es el caso de los sitios de Plazuelas (Morales et al., 2015), o el sitio de Zaragoza o Cerro de los Chichimecas (Fernández, 2016) al este y sureste en las estribaciones de la región de los Altos de Jalisco. Estas poblaciones prehispánicas que son cronológicamente parecidas es muy probable que convivieran con el sitio del Cerrito de Los Agaves, hacia el periodo Epiclásico, con los que suponemos tuvieron algún contacto relacionado con el comercio o una interacción de tipo político, religioso y/o comercial.

Por los tipos de construcciones en el sitio de Los Agaves, también es muy probable una relación con tradiciones culturales más tempranas como en los "patios hundidos" o Tradición Bajío que influyó arquitectónicamente en el sitio. La presencia de un patio cerrado y un patio hundido, lo mismo sobre la existencia del Altar Central del patio cerrado o Plaza Principal que por su gran tamaño infiere una muestra física plasmada en la arquitectura del sincretismo religioso entre diferentes tradiciones culturales.

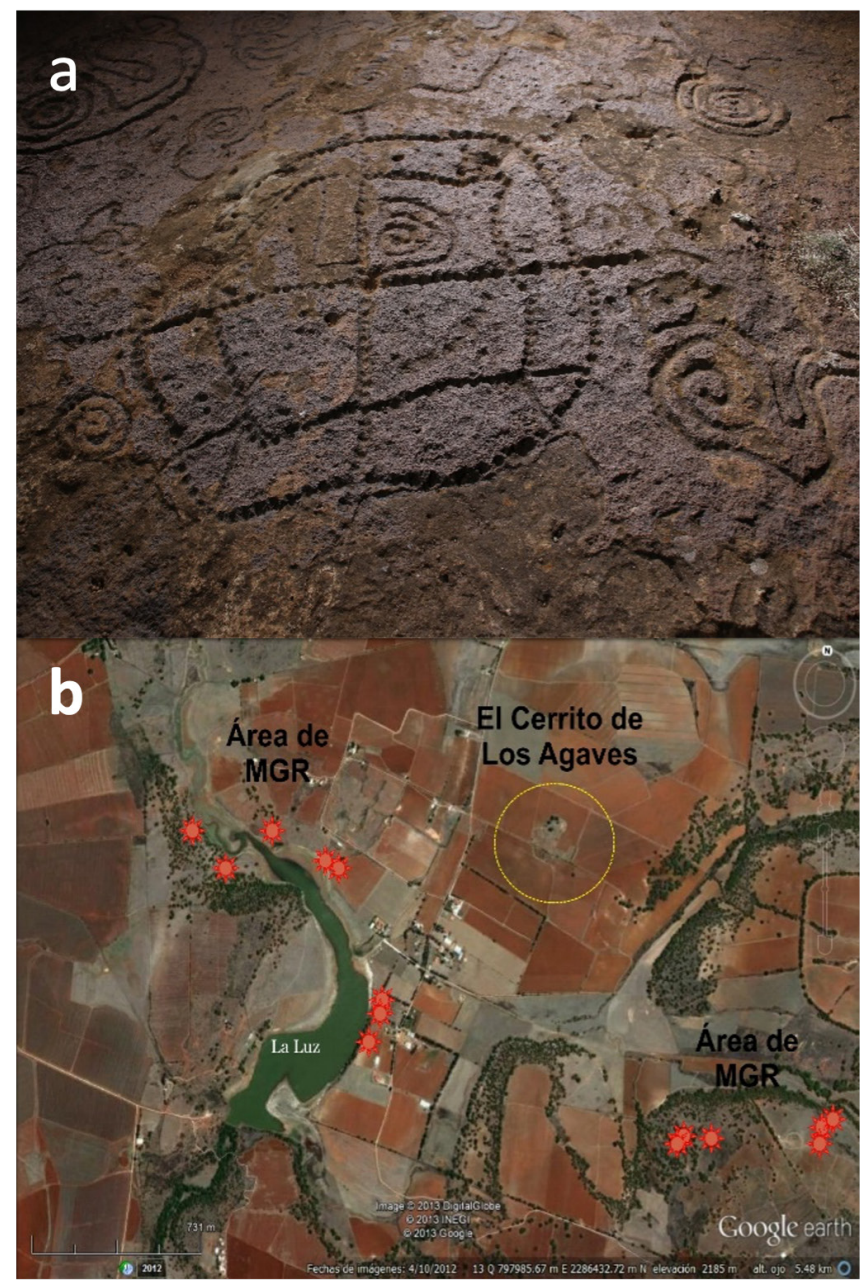

Figura 4 a) Fotografía de un fragmento del Ilamado "Planchón principal" donde se puede observar uno de los dos pecked cross que conforman el conjunto (Esparza y Rodríguez, 2016). b) Mapa de localización de los pecked cross alrededor del complejo Cerrito de Los Agaves. 


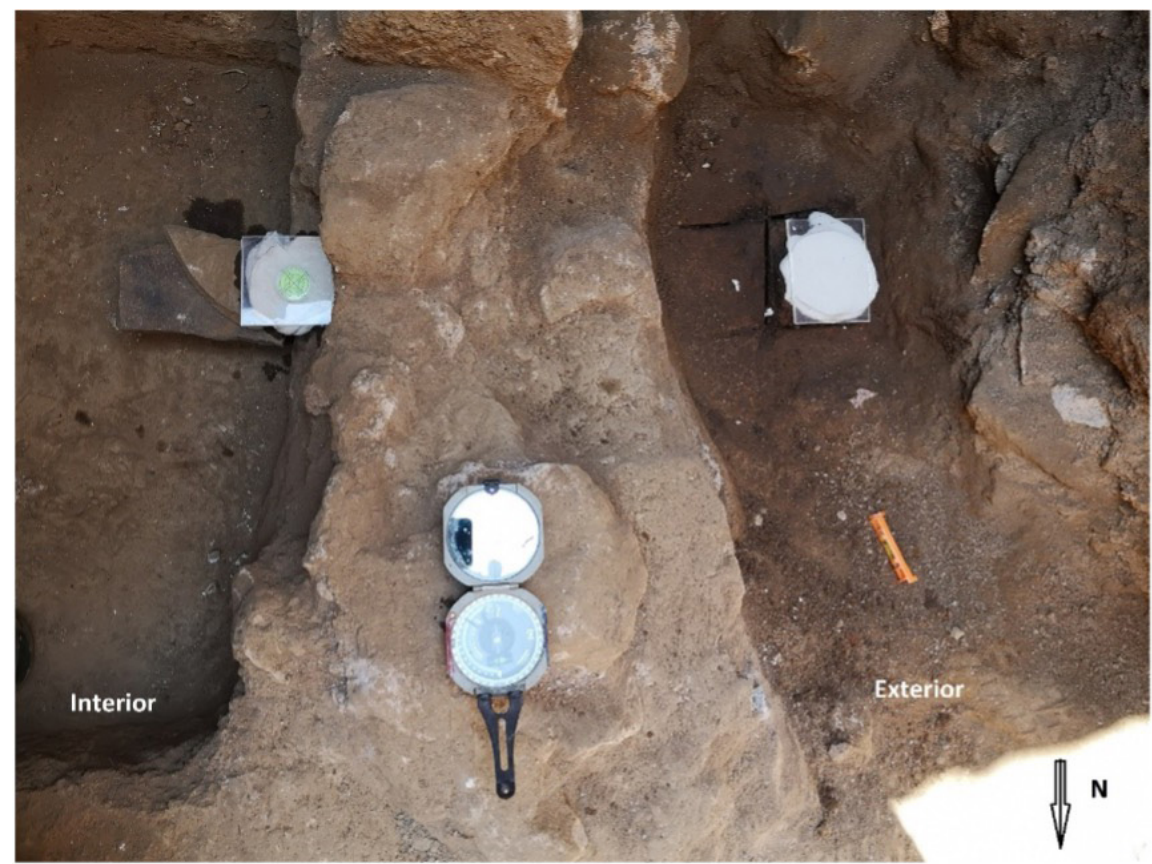

Figura 5 Detalle de la toma de muestras arqueomagnéticas orientadas dentro de Pozo S12E2.

\section{PISO EXTERIOR}
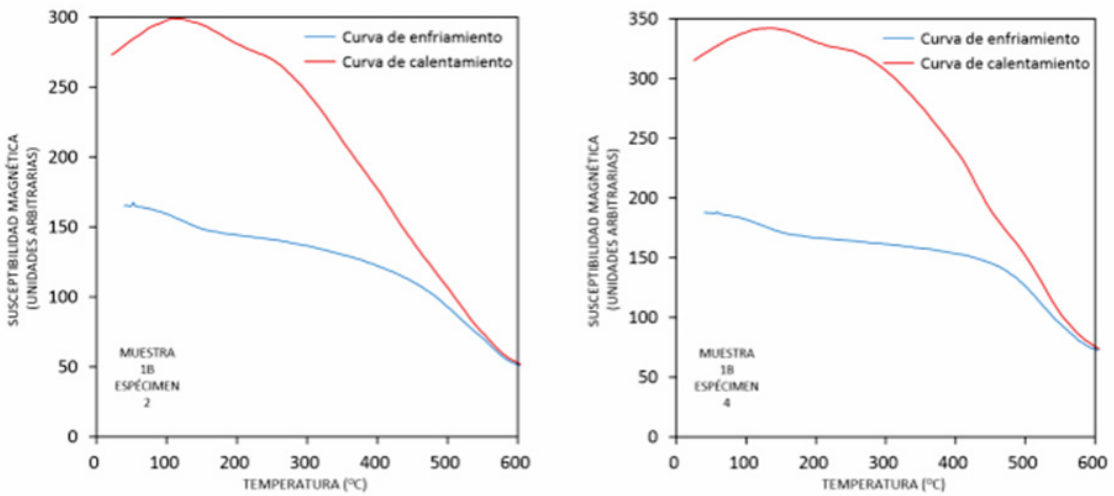

PISO INTERIOR
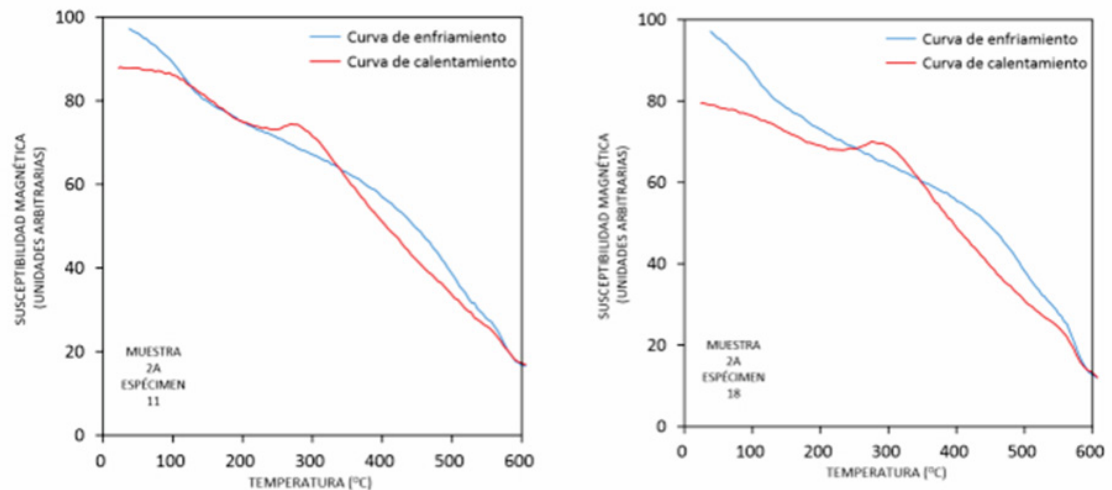

Figura 6 Registro de la susceptibilidad magnética en función de Temperatura hasta $600^{\circ} \mathrm{C}$ mediante ciclos de calentamiento y enfriamiento. 


\section{PISO INTERIOR}

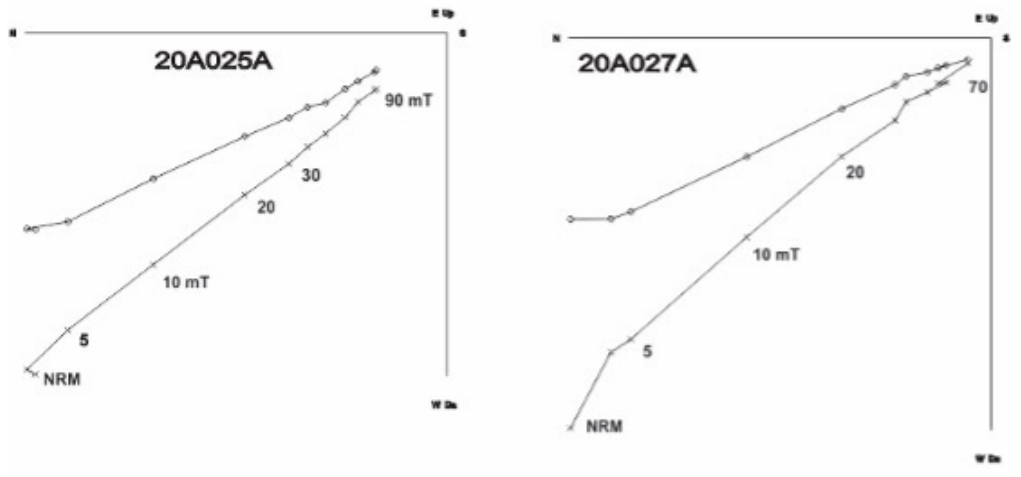

\section{PISO EXTERIOR}

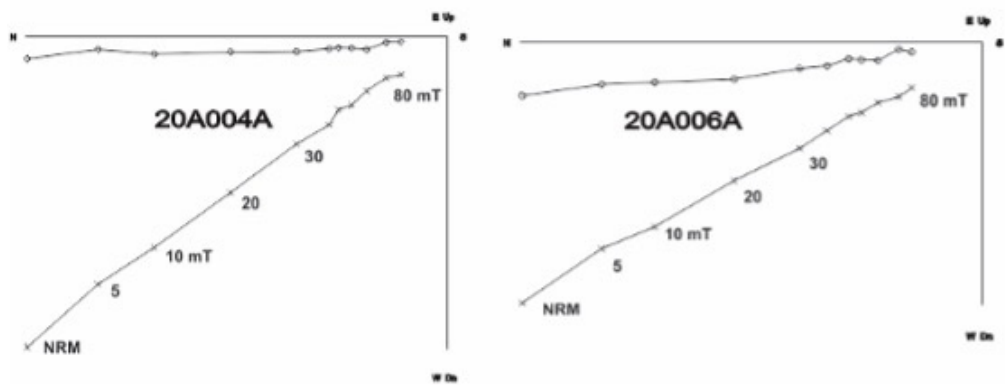

Figura 7 Diagramas ortogonales con ejemplos representativos de los procesos de desmagnetización por campos alternos. Los números indican el valor del campo aplicado en cada paso de desmagnetización hasta alcanzar los 90 miliTeslas(mT).

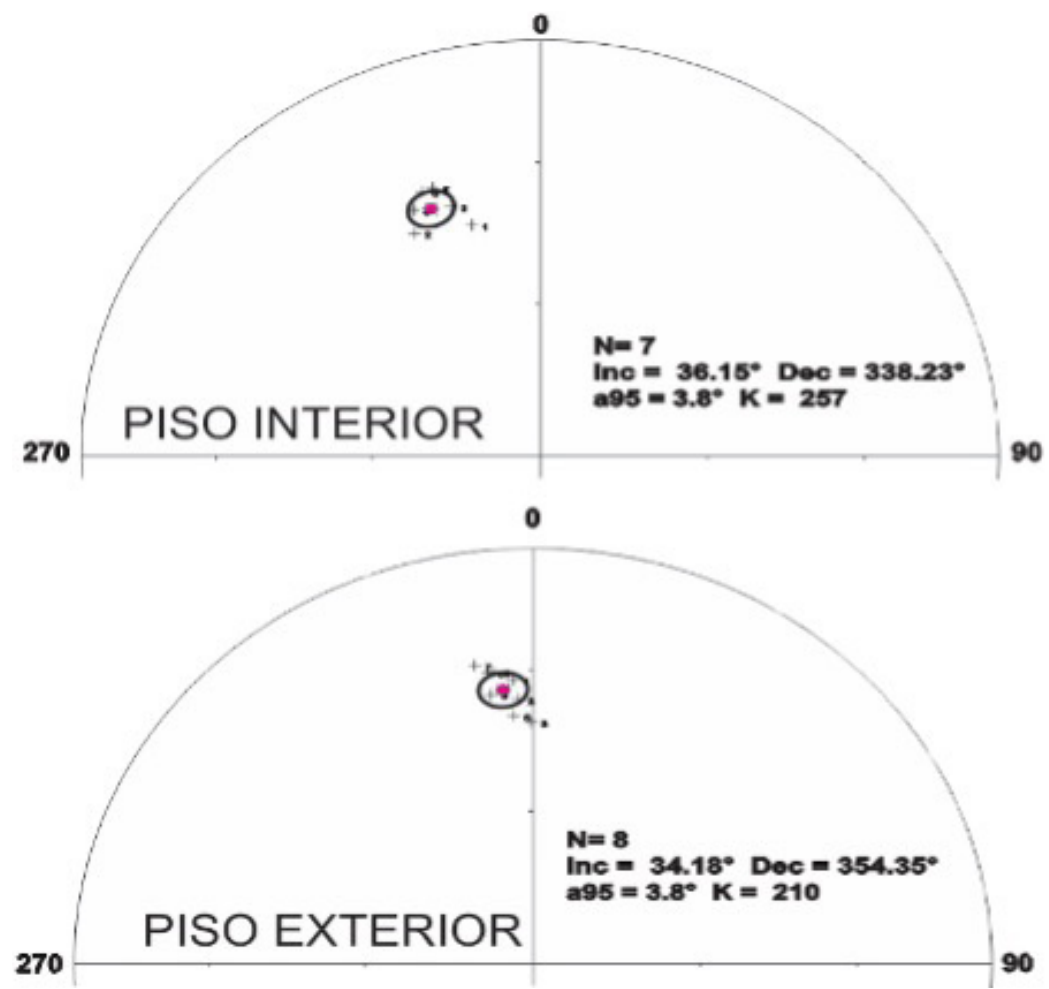

Figura 8 Diagramas de igual área con las arqueodirecciones medias (rosa) y los parámetros de precisión de la estadística de Fisher (1953) correspondientes. 

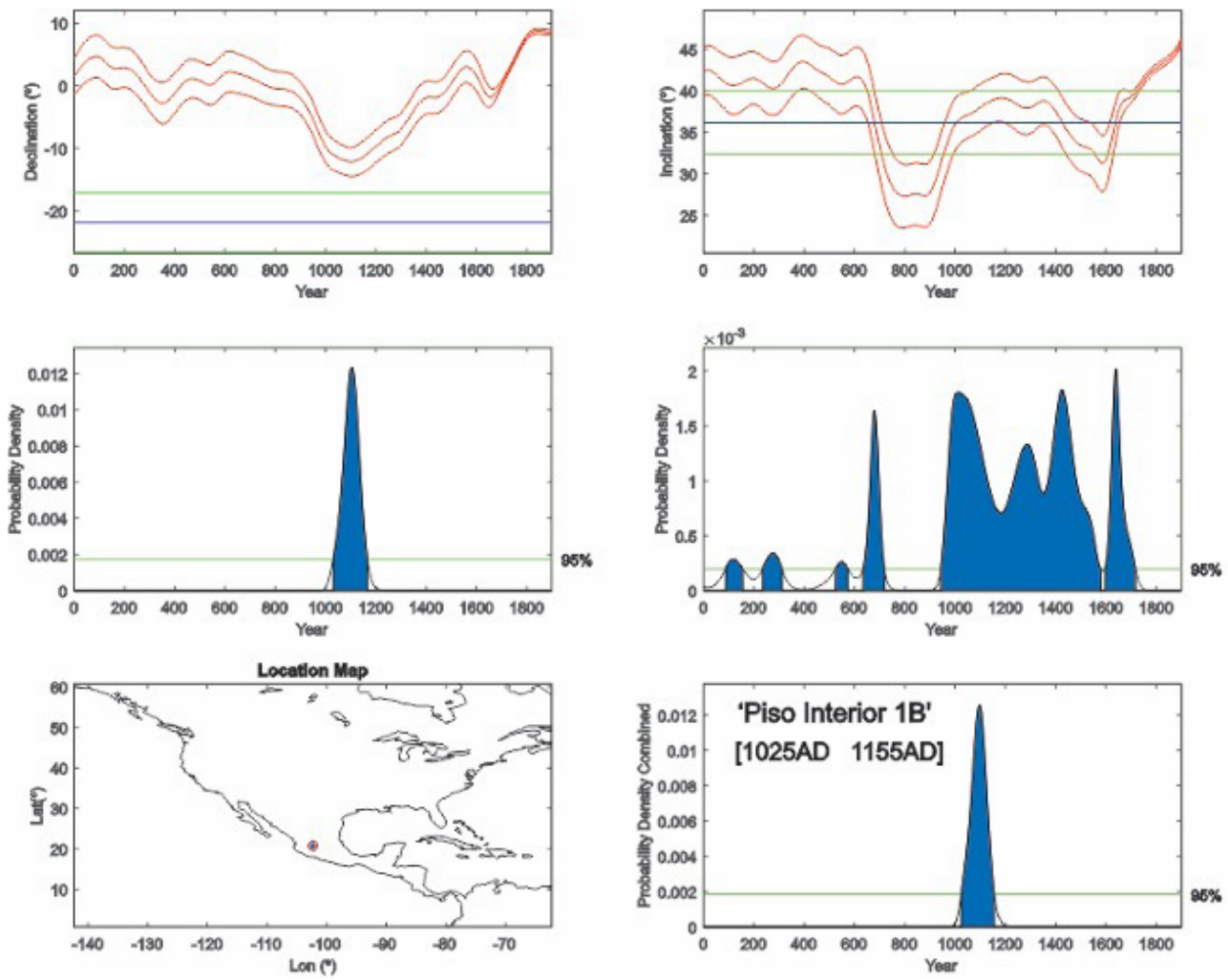

Figura 9 Datación del piso interior utilizando el modelo SHA.DIF14K (Pavón-Carrasco et al., 2011 y 2014).
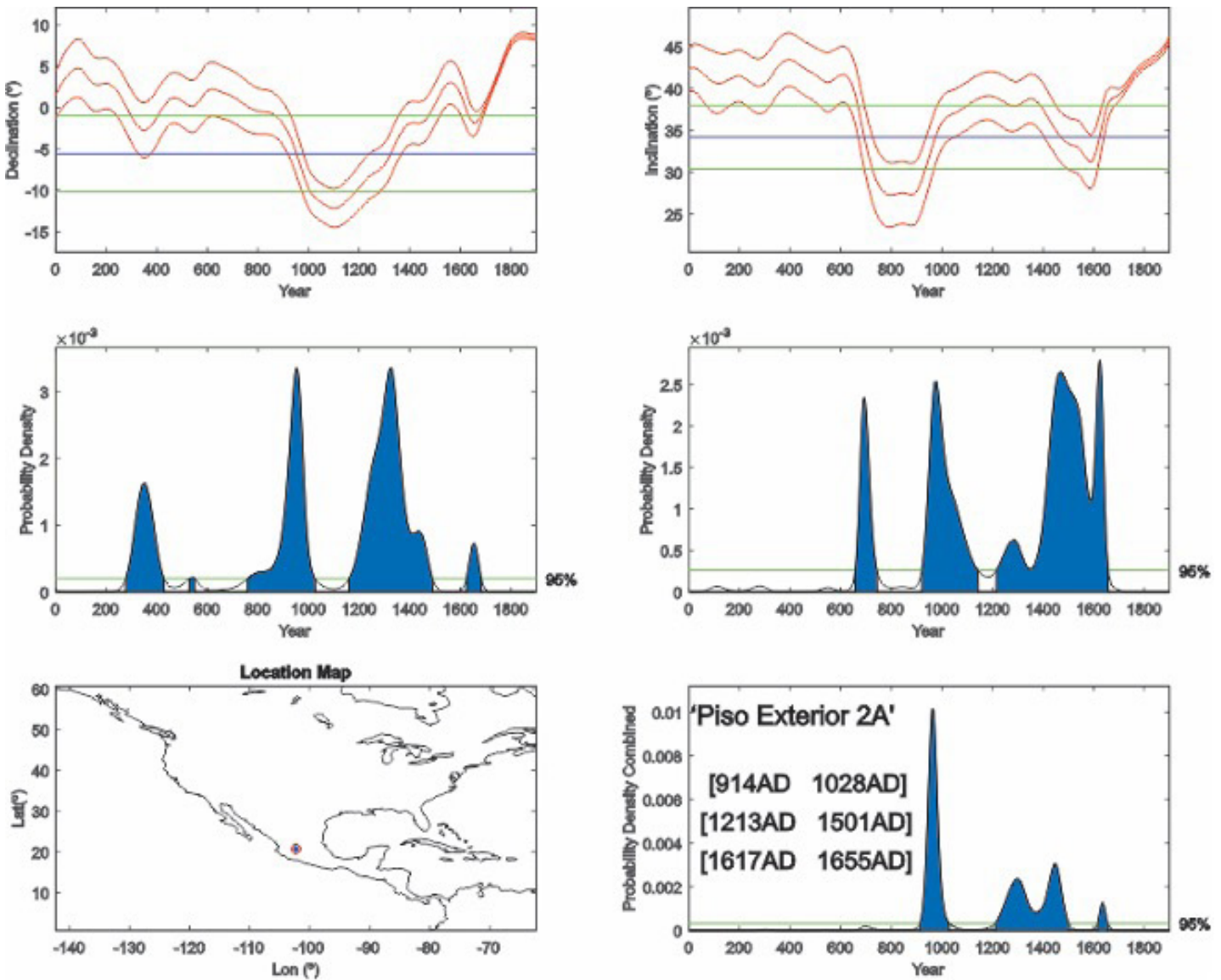

Figura 10 Datación del piso exterior utilizando el modelo SHA.DIF14K (Pavón-Carrasco et al., 2011 y 2014). 
Tabla 1. Fechamientos publicados de los Altos de Jalisco por diferentes autores. (Tomado de Porcayo, 2002 y reeditado por Esparza y Rodriguez, 2016).

\begin{tabular}{|c|c|c|}
\hline Autor & Sitio o Región & Fechamiento \\
\hline Betty Bell (1974) & Teocaltiche & 100-250 d.C. \\
\hline $\begin{array}{l}\text { Román Piña Chan y Taylor } \\
\text { (1976) }\end{array}$ & $\begin{array}{l}\text { El Cuarenta en Lagos de } \\
\text { Moreno }\end{array}$ & $\begin{array}{l}\text { Fase I: } 500-750 \text { d.C. } \\
\text { Fase II: } 750-1000 \text { d.C. }\end{array}$ \\
\hline $\begin{array}{l}\text { Beatriz Barba y Román Piña } \\
\text { Chan (1980) }\end{array}$ & $\begin{array}{l}\text { El Cerrito en el Valle de } \\
\text { Guadalupe }\end{array}$ & 600-650 a $1100-1150$ d.C. \\
\hline $\begin{array}{l}\text { Jorge Ramos, Lorenza López y } \\
\text { Carlos Santos (1992) }\end{array}$ & $\begin{array}{l}\text { San Juan de los Lagos, Unión } \\
\text { de San Antonio y Lagos de } \\
\text { Moreno }\end{array}$ & $\begin{array}{l}\text { Fase I: } 200 \text { a.C.-300 d.C. } \\
\text { Fase II. } 300-900 \text { d.C. }\end{array}$ \\
\hline Blas Castellón Huerta (1993) & Atotonilco-Arandas & 100-900 d.C. \\
\hline Alfonso Araiza (1999) & Lagos de Moreno & $\begin{array}{l}\text { Fase Lagos } 350-400 \text { a } 700-800 \\
\text { d.C. }\end{array}$ \\
\hline Antonio Porcayo (2002) & Lagos de Moreno & $\begin{array}{l}\text { Fase I: } 802-950 \text { d.C. } \\
\text { Fase II: } 950-1028 \text { d.C. }\end{array}$ \\
\hline Morales et. al., (2015) & Plazuelas & 907-997d.C. (Abandono) \\
\hline García Pimental et. al., (2020) & El Cóporo & $\begin{array}{l}\text { Fase I: } 600-1000 / 1100 \text { d.C. } \\
\text { Fase II: } 1300-1500 \text { d.C. }\end{array}$ \\
\hline
\end{tabular}

Asociado a estas características arquitectónicas, podemos observar también un patrón similar en los tipos cerámicos rescatados durante la primera temporada de excavación en el Cerrito de Los Agaves. Aunque fueron pocos los materiales diagnósticos recuperados hasta ahora, se aprecian tipos muy característicos del periodo Epiclásico como son los cajetes trípodes, algunos con dibujos incisos, las ollas con borde engargolado, así como algunos fragmentos de copas o copas pedestal (Figura 11).

La mayoría de los materiales no presentaba decoración, sin embargo, había algunos pocos con dibujos de líneas geométricas de color rojo sobre bayo y otros de rojo sobre blanco que concuerdan como lo han registrado López Mestas y Mantejano (2003) para el periodo Epiclásico entre el 600 y 900 d.C.
Por lo anterior y apoyándose también en las edades obtenidas mediante el método arqueomagnético, al parecer el periodo de ocupación principal de Cerrito de Los Agaves ocurrió entre los años 600 y 1000 de nuestra era. La presencia de petrograbados sobre todo de los llamados pecked cross encontrados en las inmediaciones del sitio, son una evidencia de relaciones con el Centro de México, en especial con Teotihuacán, si consideramos que estas evidencias aparecen en el Occidente posterior a su caída (650 d.C.), entonces existe una ocupación importante hacia estos tiempos, aunque a ciencia cierta, no hemos podido ver una relación entre el trazo del sitio de Los Agaves en relación con los pecked cross, cuestión que en Teotihuacan es muy clara y ha sido estudiada por otros autores como Aveni (2005). 
Por otro lado, las otras evidencias halladas durante las excavaciones, principalmente los derrumbes por causas naturales, así como de suelos residuales observados en el altar y en la escalinata de la estructura (Montículo Principal) permiten inferir un abandono pacífico del sitio, el cual difiere por muy poco con la destrucción y abandono de Plazuelas (Morales et al., 2015) como se puede observar en la Tabla 1, por lo que se puede establecer como un movimiento poblacional general en la región tanto del Bajío como de los Altos de Jalisco.

En la tabla 1 también se pueden observar otros fechamientos de Los Altos de Jalisco, los cuales en su mayoría son muy similares a los que tenemos para el sitio de El Cerrito de Los Agaves. Cabe mencionar que los primeras siete son por Carbono 14 y las últimas 3 son mediante datación arqueomagnética.
Siglos después de este repliegue los grupos nómadas mejor conocidos como Chichimecas ocupan de nuevo la región llevando consigo un nuevo modo de vida distinto al de los antiguos habitantes. En los Altos, los Guachichiles y Tuxcuecos subsisten explotando los recursos del medio ambiente dejados por los sedentarios e incluso en algunos lugares tienden campamentos sobre los asentamientos abandonados; a pesar de esto la evidencia arqueológica muestra que los guachichiles nunca tuvieron la misma densidad poblacional que los antiguos alteños y, de hecho, en la mayoría de los casos habitaban en los lugares más inaccesibles de la región, aunque no hemos encontrado evidencia de esta ocupación cercana al Cerrito de Los Agave. Estos grupos subsistieron un par de siglos hasta que su espíritu indómito fue sometido por los españoles (Gutiérrez, 1991).
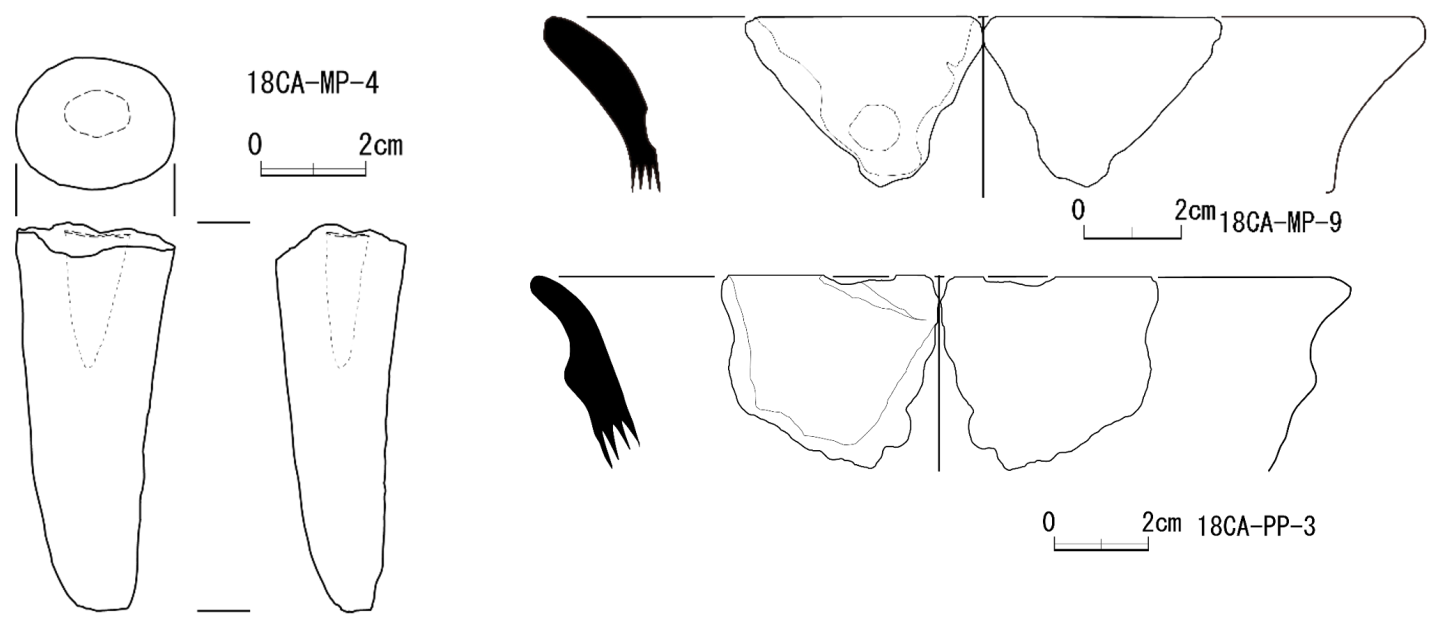

$0 \quad 2 \mathrm{~cm} \quad 18 \mathrm{CA}-\mathrm{PP}-3$
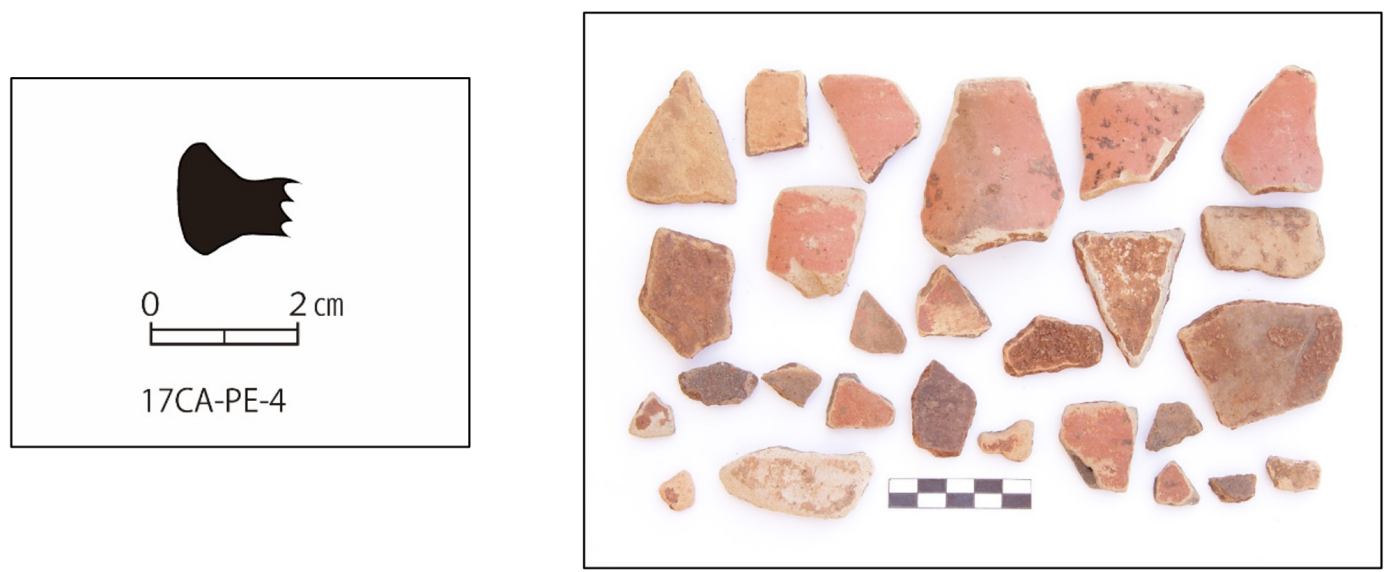

Figura 11 Se observan los materiales cerámicos de las excavaciones en Montículo Principal y Altar Central. 


\section{Agradecimientos}

Este trabajo fue apoyado por los proyectos CONACYT n. 252149 y UNAM-PAPIIT n. ${ }^{\circ}$ IN101920. M. Cervantes-Solano agradece el apoyo del proyecto CONACYT CF-2019-1761.

\section{Referencias}

Araiza, J.A., 1999, Rescate carretera Lagos de Moreno (Jal.)- San Luis Patosi ( Slp): Informe Final,Vol. 1 y 2: México, Dirección de Salvamento Arqueológico, Instituto Nacional de Antropología e Historia.

Araiza, J.A., 2000, Estudio arqueológico del Valle del río de Lagos, Jalisco: México, Escuela Nacional de Antropología e Historia, tesis de licenciatura.

Aveni, A.F., 2005, Observations on the pecked designs and other figures carved on the South platform of the pyramid of the Sun at Teotihuacan: Journal for the History of Astronomy, 36, (122), 31 - 47. https://doi. org/10.1177/002182860503600105

Barba de Piña Chan, B., Piña Chan, R., 1980, El Cerrito del Valle de Guadalupe, Jalisco, en Dahlgren, B., Navarrete, G., Ochoa, L., Serra, M.C., Sugiura, S. (org.), Homenaje a Román Piña Chan: México, Instituto Nacional de Antropología e Historia, 467-520.

Bell, B., 1974, Excavations at Cerro Encantado, Jalisco, en Bell, B. (ed.), The archaeology of West Mexico: Ajijic, Jalisco, México, Sociedad de Estudios Avanzados del Occidente de México.

Castellón, B., 1993, Cerámica de la región de Atotonilco-Arandas, Altos de Jalisco: Revista de la Coordinación Nacional de Arqueología del INAH, 2(9-10), 49-59.

Dunlop, D.J., Özdemir, Ö., 2015, Magnetizations in rocks and minerals: Treatise on geophysics, 5, 255-308. https://doi.org/10.1016/ b978-0-444-53802-4.00102-0

Dunlop D.J., Özdemir Ö., 1997, Rock magnetism: fundamentals and frontiers: Cambridge
University Press, 573 p. https://doi. org/10.1017/CBO9780511612794

Esparza, R., Rodríguez, F., 2016, El Santuario rupestre de los Altos de Jalisco. Participación comunitaria para la conservación del patrimonio cultural y natural en Jesús María, Jalisco: México, PECDA-Jalisco y El Colegio de Michoacán, A.C., 152 p.

Esparza, R., Rodríguez, F., Yoshida, T., Rétiz, M., 2018, Informe Técnico de la Cuarta Temporada del Proyecto Arqueológico Presa de la Luz, Municipio de Jesús María, Jalisco, Informe al Consejo de Arqueología del INAH (Manuscrito inédito).

Fernández, E., 2016, La región del Lerma medio como un umbral estratégico durante el epiclásico, en Augusto Montes, O., Herrejón Peredo, C. (eds), Umbrales de Michoacán. regiones fronterizas y límites territoriales: Zamora, El Colegio de Michoacán, A.C. 19-34.

Fisher, R., 1953, Dispersion on a Sphere: Proceedings of the Royal Society of London: Series A. Mathematical, Physical and Engineering Sciences, 217, 295-305. https:// doi.org/10.1098/rspa.1953.0064.

Gutiérrez, J.A. 1991, Los Altos de Jalisco. Panorama histórico de una región y de su sociedad hasta 1821: México, Consejo Nacional para la Cultura y las Artes, 549 p.

Kirschvink, J.L., 1980, The least squares line and plane and the analysis of paleomagnetic data: Geophysical Journal International, 62, 699-718. https://doi.org/10.1111/j.1365246X.1980.tb02601.x

López-Mestas Camberos, L., Montejano, M., 2003, Investigaciones arqueológicas en La Higuerita, Tala: Revista del Seminario de Historia Mexicana, 4(1), 11-33.

López, L., Montejano, M., 2009, El complejo El Grillo del centro de Jalisco: redes de intercambio y poder durante el Clásico tardío, en Williams, E., López Mestas, L., Esparza, R., (eds.), Las sociedades complejas del occidente de México en el mundo mesoamericano: Homenaje a Phil G. 
Weigand: México, El Colegio de Michoacán, 135-162.

Montejano, M.C., 2007, El Complejo el Grillo en la Porción Oriental del Valle de Atemajac, Jalisco. Desarrollo Cultural en Tonalá, Durante el Clásico Tardío-Epiclásico (450-900 d.C.), Universidad Autónoma de Guadalajara, Zapopan, Jalisco, tesis de licenciatura, $244 \mathrm{p}$.

Morales, J., Castañeda, C., Cárdenas, E., Goguitchaivili, A., 2015, Nuevas evidencias sobre la edad de abandono del sitio arqueológico Plazuelas (Guanajuato, México) mediante la datación arqueomagnética de un piso quemado: Arqueología Iberoamericana, 7(28), 40-45.

Pavón-Carrasco, F.J., Rodríguez-González, J., Osete, M.L., Miquel, J., 2011, A Matlab tool for archaeomagnetic dating: Journal of Archaeological Science, 38, 408-419. https://doi.org/10.1016/j.jas.2010.09.021.

Pavón-Carrasco, F.J., Osete, M.L., Torta, J.M., De Santis, A., 2014, A geomagnetic field model for the Holocene based on archaeomagnetic and lava flow data: Earth and Planetary Science Letters, 388, 98-109. https://doi. org/10.1016/j.epsl.2013.11.046.
Pimentel, A.G., Goguitchaichvili, A., Torreblanca, G., Cejudo, R. Kravchinsky, V., García, R., Morales, J., Cervantes, M., 2020, The memory of fire in El Coporo (Northern Mesoamerica): Apogee and abandonment: Journal of Archaeological Science: Reports, 30, 102274. https://doi.org/10.1016/j. jasrep.2020.102274

Piña Chan, R., Taylor, J., 1976, Cortas excavaciones en el Cuarenta, Jalisco: México, Departamento de Monumentos Prehispánicos, INAH.

Porcayo, A., 2002, Testimonio de una colonización efimera. Historia pre chichimeca de Lagos de Moreno, Jalisco: México, CONAGULTAINAH, Archivo Histórico Municipal.

Prévot, M., Mankinen, E.A., Grommé, S., Lecaille, A., 1983, High paleointensities of the geomagnetic field from thermomagnetic studies on rift valley pillow basalts from the Mid-Atlantic Ridge: Journal of Geophysical Research: Solid Earth, 88(B3), 2316-2326. https://doi.org/10.1029/JB088iB03p02316.

Ramos, J., López, L., Santos, C., 1992, Proyecto Altos de Jalisco: Jalisco, México, Archivo del Centro INAH Jalisco, Guadalajara, Informe técnico temporada. 